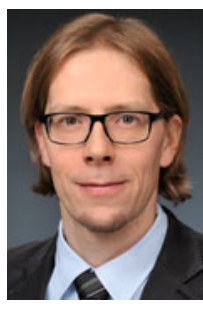

Nils Helge Schebb

1998-2006 Studium der

Lebensmittelchemie, Toxikologie und Biochemie an den Universitäten Münster und Karlsruhe. 2006-2009 Promotion in Analytischer Chemie an der Universität Münster (Prof. Dr. U. Karst). 2009-2011 Postdoc an der University of California, Davis, USA (Prof. Dr. B. D. Hammock). Seit 2011 unabhängige Forschung zur biologischen Aktivität von Lebensmittelinhaltstoffen als Habilitand von Prof. Dr. P. Steinberg an der Tierärztlichen Hochschule Hannover.

$10.1007 / \mathrm{s} 12268-014-0488-0$

(C) Springer-Verlag 2014

Triclocarban (TCC) wird Seifen und anderen Kosmetika als antibakterieller Wirkstoff zugesetzt, obwohl neue Studien auf eine deutliche Bioakkumulation in aquatischen Systemen und eine hohe biologische Aktivität in Säugetieren hinweisen. So ist TCC beispielsweise ein nanomolarer Inhibitor der löslichen Epoxidhydrolase, einem zentralen Enzym der Arachidonsäurekaskade (u. a. Regulation von Entzündung, Fieber und Schmerz).

Beim Duschen und Baden mit TCC-haltiger Seife kommt es zur Deposition des lipophilen TCCs auf der Haut, und ein kleiner, aber relevanter Anteil durchdringt die dermale Barriere. Das absorbierte TCC unterliegt einem ausgeprägten Fremdstoffmetabolismus, insbesondere findet eine oxidative Umsetzung durch Cytochrom-P450-Monooxygenasen (CYP) statt.

In den vorgestellten Arbeiten haben wir untersucht, ob es im TCC-Metabolismus zu einer metabolischen Aktivierung kommen kann. Hierzu wurden Inkubationen von spontan immortalisierten Keratinozyten (SIK) mit TCC (zwei Mikromol pro Liter) durchgeführt, jeweils mit und ohne Präinduktion durch den Arylhydrocarbon(Ah)-Rezeptor-Agonisten 2,3,7,8-Tetrachlordibenzodioxin (TCDD). TCC wurde schnell aus dem Medium absorbiert,

\title{
Sanofi-Aventis-Preis 2014 (Kurzvortrag) Metabolische Aktivierung von Triclocarban
durch Cytochrom P450 1A1
}

NILS HELGE SCHEBB

INSTITUT FÜR LEBENSMITTELTOXIKOLOGIE UND CHEMISCHE ANALYTIK, TIERÄRZTLICHE HOCHSCHULE HANNOVER

und es resultierte eine zelluläre Konzentration von 2,04 \pm 0,27 Nanomol pro Milligramm Protein. Dabei wurde ein Teil des TCCs oxidativ zu hydroxylierten Metaboliten umgesetzt, wobei deutlich höhere Metabolitkonzentrationen nach TCDD-Behandlung gebildet wurden. Unter Einsatz von ${ }^{14} \mathrm{C}$-markiertem TCC konnten wir auch die Bildung von Proteinaddukten belegen $(23 \pm 2$ Pikomol pro Milligramm Protein) [1]. Ebenso führten mikrosomale Inkubationen mit CYP1 A1, einer durch TCDD induzierbaren CYP, zu TCC-Glutathionaddukten. Die Struktur der Glutathionaddukte und ihr Bildungsweg wurde mittels hochauflösender Massenspektrometrie detailliert charakterisiert [2]. Die Ergebnisse deuten darauf hin, dass TCC durch oxidative Dehalogenierung und Oxidation zu einem reaktiven $p$-Chinonimin aktiviert wird (Abb. 1). Aus toxikologischer Sicht ist die Bildung von reaktiven Metaboliten nicht direkt gleichzusetzen mit einer schädigenden Wirkung auf die Gesundheit. Allerdings ist dies oft ein Alarmsignal, da reaktive Metaboliten beispielsweise zu Kontaktallergien oder DNASchäden bzw. Mutationen führen können.

Nachdem TCC seit mehr als 50 Jahren in hoher Konzentration in Kosmetika (bis zu 1,5 Prozent) eingesetzt wird, war es überraschend, dass die Bildung von reaktiven Metaboliten über so lange Zeit unerkannt blieb.

\section{Literatur}

[1] Schebb NH, Buchholz B, Hammock BD et al. (2012) Metabolism of the antibacterial triclocarban by human epidermal keratinocytes to yield protein adducts. J Biochem Mol Toxic 26:230-234

[2] Schebb NH, Muvvala JB, Morin D et al. (2014) Metabolic activation of the antibacterial agent, triclocarban, by cytochrome P450 1A1 yielding glutathione adducts. Drug Metab Dispos 42:1098-1102

Korrespondenzadresse: Dr. Nils Helge Schebb Institut für Lebensmitteltoxikologie und Chemische Analytik Tierärztliche Hochschule Hannover Bischofsholer Damm 15 D-30173 Hannover Tel.: 0511-856-7780 Fax: 0511-856-7409 schebb@wwu.de http://schebb-web.de<smiles>CC(C)(C)C(=O)Nc1ccc(N=C(NC(=O)Nc2ccc(O)c(Cl)c2)Nc2ccc(Cl)c(Cl)c2)cc1</smiles>

A Abb. 1: Vorgeschlagener Mechanismus der Bildung von Triclocarban(TCC)-Glutathionaddukten. Ox: Oxidation; GSH: Glutathion. 